\title{
Envolvimento dos estudantes no ensino superior: um estudo com a escala
}

\author{
EAE-E4D
}

\section{Students' engagement in third level education: a study with the scale SES- 4DS}

\author{
Filomena Covas*, Feliciano Veiga ** \\ *Instituto Politécnico de Lisboa ** Instituto de Educação da Universidade de Lisboa
}

\begin{abstract}
Resumo
Enquadramento Conceptual: Considera-se o Envolvimento do Aluno na Escola um constructo quadridimensional, abrangendo as dimensões; cognitiva, afetiva, comportamental e agenciativa. Objetivo: Analisar as qualidades psicométricas da adaptação para contexto de ensino superior, da escala de Envolvimento dos Alunos na Escola: uma Escala Quadri-Dimensional - EAE-E4D. Metodologia: Quantitativa. Amostra de 715 estudantes do Ensino Superior. Resultados: Estudo das propriedades psicométricas da escala EAE-E4D através da análise factorial e da avaliação da consistência interna. Os resultados reproduzem valores semelhantes aos da escala original. Conclusões: O questionário EAE-E4D apresenta boas qualidades psicométricas e pode, também, ser usado com estudantes do Ensino Superior.

Palavras-chave: envolvimento do aluno na escola, ensino superior, EAE-E4D, qualidades psicométricas
\end{abstract}

\begin{abstract}
Conceptual Framework: Student Engagement in School is considered as a four-dimensional construct, comprising the cognitive, affective, behavioural, and agentic dimensions. Objective: To analyse the psychometric properties of the adaptation of the scale Students' Engagement in School: Four-Dimensional Scale - SES4DS to a higher education context. Methodology: Quantitative research. Sample of 715 higher education students. Results: Evaluation of the psychometric qualities of the SES-4DS through factor analysis and assessment of the internal consistency. The results reveal similar values to the original scale's. Conclusion: The SES-4DS questionnaire shows good psychometric properties and may also be used with higher education students.

Keywords: student engagement in school, higher education, SES-4DS, psychometric qualities
\end{abstract}

\section{Enquadramento conceptual}

O envolvimento do aluno na escola é considerado um conceito complexo e multifacetado que tem sido alvo de considerável atenção, na literatura especializada em educação, nos últimos 30 anos. As primeiras contextualizações do conceito de envolvimento foram realizadas no âmbito das teorias do desenvolvimento do estudante, estabelecendo uma forte ligação deste conceito a uma série de outras dimensões educacionais, tais como os resultados positivos no desenvolvimento académico dos estudantes, incluindo aspectos como a participação, a satisfação, a persistência, a realização académica e o compromisso social (Astin, 1984). Estas perspectivas estavam essencialmente associadas aos comportamentos observáveis dos alunos como é o caso do modelo de participação-identificação apresentado por Finn (1989), no qual as dimensões académica, social e cognitiva integravam a dimensão comportamental que permitia caracterizar o envolvimento dos alunos.

Mais recentemente, o envolvimento tem sido definido por alguns autores como um estado mental positivo que se caracteriza por vigor, dedicação e absorção nas atividades, refere-se à manifestação simultânea de concentração, interesse e prazer na realização da atividade, o que significa que se caracteriza também por fatores emocionais e afetivos, para além de cognitivos e comportamentais (Fredricks, Blumenfeld, \& Paris, 2004; Schaufeli, Salanova, Bakker, \& Gonzales-Roma, 2002; Veiga, Festas, et al., 2012).

$\mathrm{Na}$ literatura anglo saxónica o constructo de envolvimento frequentemente assume designações pouco diferenciadas tais como: Engagement; Engagement in Schoolwork; Academic Engagement; School Engagement; Student Engagement; Student Engagement in Academic Work; e Student Engagement in/with School (Appleton, Christenson, \& Furlong, 2008, pp. 371-372). No intuito de clarificar as diferentes terminologias alguns autores referem a sua preferência pela utilização da expressão de Student Engagement in/with School (SES), justificando que esta designação inclui de forma mais completa as atividades académicas relacionadas com os alunos e que podem acontecer tanto no interior da escola, como nos contextos externos à escola onde se inclui a família e a comunidade em geral (Appleton et al., 2008). No presente estudo, também se optou por esta última designação apresentada na sua tradução - Envolvimento dos Alunos na Escola (EAE)

O EAE, pode então ser definido como um fenómeno que acontece quando se estabelece uma sintonia entre os interesses individuais e os interesses da escola ou como uma vivência centrípeta do aluno à escola (Veiga, Festas, et al., 2012; Veiga, Galvão, et al., 2012). Sendo assim, o conceito de envolvimento é operacionalizado 
valorizando o nível de motivação, e de comprometimento dos alunos, com a escola e com a aprendizagem, e tem sido estudado como uma estrutura organizadora que envolve o sentimento de pertença à escola, a participação, a motivação e a auto eficácia (Fredricks et al., 2004; Veiga, Reeve, Wentzel, \& Robu, 2014). O EAE é considerado maleável e aberto às influências construtivas do contexto escolar, como o apoio dos professores, de colegas e/ou de outros agentes significativos (Reeve, 2012). Esta característica de ser sensível às práticas escolares, é considerado um dos aspetos fundamentais do constructo de envolvimento (Finn \& Zimmer, 2012).

Historicamente falando, foi a partir do estudo da motivação dos estudantes que se começou a entender, ainda que de forma pouco explícita, a qualidade da adaptação e do investimento dos estudantes na escola (Brophy, 1983). Muitas definições atuais de envolvimento continuam explicitamente relacionadas com atividades e trabalhos académicos. Por essa razão, é importante atender à relação entre envolvimento e motivação. A motivação no contexto escolar também se caracteriza como um constructo multidimensional e está associado a uma série de conceitos (e.g., competência pessoal, autoeficácia, auto-regulação) (Pintrich, 2003). São sobretudo as teorias cognitivas da motivação que destacam o estudo das crenças pessoais, sobre as causas, valores e emoções do individuo, às quais o próprio atribui a responsabilidade das suas experiências de sucesso ou de fracasso escolar. Estas crenças pessoais desempenham assim, uma função mediadora no comportamento e constituem uma forte influência no processo motivacional (B. Weiner, 1985).

Alguns aspectos que dificultam a distinção entre motivação e envolvimento estão relacionados com o facto de os dois conceitos serem influenciados pelo contexto (Reschly \& Christenson, 2012). Contudo, existe algum consenso em pensar que envolvimento e motivação surgem como constructos separados, mas significativamente relacionados na medida em que a motivação subentende uma direção, intensidade e qualidade da energia, enquanto o envolvimento é a manifestação em ação dessa mesma energia motivacional. Sendo assim a motivação torna-se necessária ao envolvimento uma vez que este é dirigido por processos motivacionais, mas não é condição suficiente (Reschly \& Christenson, 2012; Skinner, Kindermann, \& Furrer, 2008; Veiga, Festas, et al., 2012). Neste mesmo sentido, existem autores que defendem que o conceito de motivação deve ser integrado no próprio constructo de envolvimento (Fredricks et al., 2004). A noção de causalidade recíproca entre os dois constructos tem sido defendida na teoria da autodeterminação que se enquadra numa perspectiva motivacional do envolvimento (Reeve, 2012).

Existem diversas perspectivas em relação às dimensões que caracterizam o envolvimento. Podem variar de uma a quatro dimensões (Veiga et al., 2014). Entre vários autores, constam repetidamente três dimensões no constructo de envolvimento: afectiva, com o intuito de poder vir a considerar-se também comportamental e cognitiva. Mais recentemente é considerada também a dimensão agenciativa (agentic) que integra os aspectos do envolvimento associados á contribuição proactiva, intencional e construtiva do aluno no seu próprio processo de aprendizagem (Reeve, 2013; Reeve \& Tseng, 2011; Veiga, 2013; Veiga et al., 2014). A diversidade de concepções teóricas inclui a variedade das combinações possíveis entre as dimensões identificadas, refletida na quantidade de instrumentos construídos para avaliar o EAE (Fredricks et al., 2011; Fredricks \& McColskey, 2012; Veiga et al., 2014).

A perspectiva do presente estudo considera o EAE como um constructo quadri-dimensional na continuidade do estudo de Veiga (2013), que consistiu na elaboração da escala - Envolvimento dos Alunos na Escola: Uma Escala Quadridimensinal (EAE- E4D) - e na sua validação com alunos portugueses dos $6^{\circ}, 7^{\circ} 9^{\circ} \mathrm{e}$ $10^{\circ}$ anos de escolaridade. De acordo com o autor da escala, a mesma é constituída por 20 itens que avaliam o envolvimento em 4 dimensões: cognitiva, afetiva, comportamental e agenciativa. Os itens da dimensão cognitiva relacionam-se com o investimento pessoal do aluno através das estratégias metacognitivas e auto reguladoras que constrói e utiliza para compreender e relacionar os assuntos abordados nas aulas (Appleton et al., 2008; Fredricks et al., 2004; Veiga, Festas, et al., 2012). Os itens da dimensão afectiva estão relacionados com as emoções experimentadas pelos alunos na sala de aula e na escola em geral. Envolvem questões ligadas à vinculação com a escola, à forma como o aluno se sente integrado, ao interesse que tem pela escola, incluindo as atitudes que tem para com os colegas, com os professores e com a própria aprendizagem (Appleton et al., 2008; Veiga, Festas, et al., 2012). Os itens da dimensão comportamental estão relacionados com as regras formais e informais, da escola, referentes à dimensão cívica de participação responsável que podem interferir com a aprendizagem. A sua operacionalização consiste na observação das condutas e ações dos alunos em relação às diversas tarefas que integram as responsabilidades escolares. (Appleton et al., 2008; Fredricks et al., 2004; Trowler, 2010; Veiga, Festas, et al., 2012). Os itens da dimensão agenciativa relacionam-se com um processo intencional e proactivo dos alunos no âmbito da construção do seu próprio processo de aprendizagem. Os alunos tentam personalizar e enriquecer o que está a ser aprendido através de uma inter-relação entre o próprio conteúdo específico da aprendizagem e as circunstâncias pessoais e contextuais, modificando e apropriando-se do que é realmente aprendido. Inclui não apenas uma determinada reação à tarefa, com maior ou menor persistência e satisfação, mas sobretudo uma dinâmica interna na qual o aluno recria o que aprendeu potenciando a sua liberdade de ação decorrente dessa aprendizagem que é experimentada como significativa (Reeve \& Tseng, 2011; Veiga, 2013).

No presente estudo (inserido num outro mais amplo, Cf. Nota) aplicou-se a escala de EAE-E4D em estudantes portugueses de ensino superior politécnico, adequada a sua utilização a este nível de ensino. 


\section{Metodologia}

\section{Amostra}

A amostra foi constituída por 715 estudantes dos quais $364(50,9 \%)$ pertencem ao sexo feminino e 351 $(49,1 \%)$ ao sexo masculino. Quanto às idade variaram entre os 18 e os 65 anos. A média de idade situou-se em $26,14$ anos ( $D P=9,021)$, mais de metade dos estudantes $(66,9 \%)$ tem menos de 26 anos. A amostra integra estudantes dos vários anos das licenciaturas, $39 \%$ do $1^{\circ}$ ano, $24,2 \%$ do $2^{\circ}$ ano e $36,8 \%$ dos $3^{\circ}$ e $4^{\circ}$ anos.

\section{Instrumento}

A EAE-E4D foi validada por Veiga (2013) a partir das respostas de 679 alunos portugueses (291 do sexo masculino e 388 do sexo feminino) a frequentar os $6^{\circ}$, $7^{\circ}, 9^{\circ}$ e $10^{\circ}$ anos de escolaridade em diferentes zonas do país. Esta escala contém 20 itens, de resposta tipo Likert, que pode variar entre 1 (total desacordo) e 6 (total acordo). Cada uma das dimensões do envolvimento é avaliada através de um conjunto de 5 itens. Para os diferentes grupos de alunos, os valores da consistência interna da escala ( Alfa de Cronbach) variaram entre o valor máximo de 0.870 para a dimensão agenciativa e o valor mínimo de 0.689 para a dimensão comportamental (Veiga, 2013). O autor realizou um estudo de análise fatorial de componentes principais com rotação varimax, sem especificar o número de fatores a extrair e obteve 4 fatores que explicaram $57.912 \%$ da variância total, com $11.838 \%$ na dimensão cognitiva (autovalor=2.368), $14.843 \%$ na dimensão afetiva (autovalor=2.969), 6.759\% na dimensão comportamental (autovalor=1.352) e $24.427 \%$ na dimensão agenciativa (autovalor=4.894).

Sendo a EAE-E4D originalmente validada para alunos de um contexto escolar não superior, resolveu-se proceder a algumas adaptações de linguagem a partir da realização de um estudo preliminar. No âmbito deste estudo, procedeu-se à análise da frequência das respostas a cada item, uma vez que este método permite avaliar a pertinência do conteúdo de alguns itens (Tuckman, 2012). Especificamente os itens, cujas respostas apresentaram uma elevada homogeneidade centradas num dos polos da escala de resposta, foram: o item 13 - "Perturbo a aula propositadamente" e o item 14 - "Sou mal-educadola com o professor". Sendo a percentagem de resposta, nos dois itens, superior a $92 \%$ na escolha de "Total desacordo", repensou-se a pertinência do conteúdo destes itens no âmbito do contexto de ensino superior, já que os estudantes neste contexto de ensino têm um estatuto mais responsável relativamente às suas escolhas e por isso é possível que, inerentemente, possam ter um comportamento mais adequado às normas da sala de aula. No mesmo sentido constatou-se num estudo sobre as qualidades psicométricas da EAE-E4D em estudantes da Universidade dos Açores, que os itens 13 e 14 foram eliminados por estarem a prejudicar a consistência interna do instrumento (Fernandes, Caldeira, Silva, \& Veiga, 2016). Pelas razões apresentadas, considerou-se pertinente encontrar novas formulações, mais adaptadas ao contexto de ensino superior, dos itens 13 e 14 da dimensão comportamental (cf. Tabela 1).

A maioria dos itens da EAE-E4D estão formulados no sentido positivo, contudo existem 7 itens $(6,10,11,12$, 13,14 e 15) que estão formulados de forma negativa pelo que antes de iniciar a análise dos dados, se deve proceder à inversão da pontuação das respostas a estes itens. Assim, na escala em geral, as pontuações mais elevadas corresponderão a um maior envolvimento na escola.

\section{Procedimento}

Ao longo do processo desta pesquisa procurou-se respeitar os princípios éticos fundamentais que dizem respeito aos cuidados a ter com a proteção dos participantes, o seu consentimento informado, a confidencialidade, a privacidade e a proteção de recolha de dados.

Foi inicialmente realizado um estudo preliminar com uma amostra de 63 estudantes do ensino superior, este primeiro estudo teve como finalidade analisar as características que foram consideradas essenciais para equacionar a melhoria da formulação dos itens do instrumento relativamente à objetividade da informação que se pretendia recolher. Foi realizado um segundo estudo com uma amostra de 715 estudantes do Ensino Superior Politécnico da região de Lisboa, abrangendo estudantes de quatro escolas e dos vários anos dos cursos de Licenciatura.

Os dados foram recolhidos através de uma versão online, criada com o software Limesurvey e a recolha das respostas dos estudantes decorreu entre março e maio de 2016. Os dados recolhidos foram diretamente exportados para uma base de dados do software de análise estatística SPSS, versão 23.

Os procedimentos estatísticos utilizados foram, em parte, semelhantes aos utilizados no estudo desenvolvido por Veiga (2013): procedeu-se à análise estatística descritiva para a caracterização geral da amostra; realizou-se uma análise fatorial de componentes principais com rotação varimax para estudar a validade interna da escala; e analisou-se a consistência interna da escala total e das respectivas dimensões através da análise dos valores do Alfa de Cronbach.

\section{Resultados}

\section{Estudo da análise factorial}

A análise da estrutura dos itens da EAE-E4D foi efectuada através da análise factorial exploratória de componentes principais com rotação varimax sem especificar o número de fatores a extrair. Os factores comuns retidos foram os que apresentaram um autovalor inicial superior a 1 (Martins, 2011).

A análise factorial convergiu para uma solução com 4 componentes principais que explicaram $61.140 \%$ da variância total. Como referido anteriormente, no estudo da escala original a variância total explicada pelos 4 fatores foi de 57.912\% (Veiga, 2013). 
O fator afetivo explicou $26.804 \%$ da variância total (autovalor inicial=5.361); o fator agenciativo explicou $15.311 \%$ da variância total (autovalor inicial=3.062); o factor comportamental explicou $12.240 \%$ da variância total (autovalor inicial=2.448), e o factor cognitivo explicou $6.786 \%$ da variância total (autovalor inicial=1.357). A saturação dos itens (>0.60) em cada uma das componentes principais pode ser observada na Tabela 1. A primeira componente principal integra os itens relacionados com a dimensão afectiva (Afe), a segunda componente os itens relacionados com a dimensão agenciativa (Age), a terceira integra os itens relacionados com a dimensão comportamental (Com) e os últimos 5 itens correspondem à dimensão cognitiva (Cog). O resultado reproduz os 4 fatores obtidos por Veiga (2013), embora os valores para as dimensões sejam algo diferentes.

Tabela 1.

Resultados da análise factorial: EAE-E4D

\begin{tabular}{|c|c|c|c|c|}
\hline \multirow[b]{2}{*}{ Itens } & \multicolumn{4}{|c|}{ Componentes } \\
\hline & Afe & Age & Com & $\operatorname{Cog}$ \\
\hline 08. A minha escola é um lugar onde me sinto integrado/a. & .892 & & & \\
\hline 09. A minha escola é um lugar onde me parece que os outros gostam de mim. & .858 & & & \\
\hline 07. A minha escola é um lugar onde faço amigos com facilidade. & .837 & & & \\
\hline 10. A minha escola é um lugar onde me sinto só. & .829 & & & \\
\hline 06. A minha escola é um lugar onde me sinto excluído/a. & .806 & & & \\
\hline 19. Durante as aulas, intervenho para exprimir as minhas opiniões. & & .850 & & \\
\hline 17. Falo com os meus professores sobre aquilo de que gosto e não gosto. & & .839 & & \\
\hline 18. Comento com os meus professores, quando alguma coisa me interessa. & & .822 & & \\
\hline 20. Faço chegar as minhas sugestões de melhoria aos professores. & & .788 & & \\
\hline 16. Durante as aulas, participo colocando questões. & & .725 & & \\
\hline 13. Perturbo as aulas com conversas que em nada têm a ver com os assuntos de estudo.* & & & .778 & \\
\hline 15. Estou distraído/a nas aulas. & & & .705 & \\
\hline 14. Tenho comportamentos que revelam falta de consideração pelos meus professores.* & & & .691 & \\
\hline 12. Falto às aulas estando na escola. & & & .686 & \\
\hline 11. Falto à escola sem uma razão válida. & & & .640 & \\
\hline 02. Procuro relacionar o que aprendo numa disciplina com o que aprendi noutras. & & & & 678 \\
\hline 04. Quando estou a ler, procuro compreender o significado daquilo que o autor quer transmitir. & & & & .656 \\
\hline 05. Revejo regularmente os meus apontamentos, mesmo que um teste ainda não esteja próximo. & & & & .655 \\
\hline 01. Quando escrevo os meus trabalhos, começo por fazer um plano para o texto a redigir. & & & & .625 \\
\hline $\begin{array}{l}\text { 03. Passo muito do meu tempo livre à procura de mais informação sobre tópicos discutidos nas } \\
\text { aulas. }\end{array}$ & & & & 618 \\
\hline
\end{tabular}

Nota: * Itens com formulação adaptada ao ensino superior, algo diferentes da escala original.

\section{Estudo da consistência interna}

Os valores de consistência interna da EAE-E4D variaram entre um mínimo de 0.758 (razoável) na dimensão Comportamental a um máximo de 0.906 (elevado) na dimensão Afectiva. Na Tabela 2 indicam-se também os valores de consistência interna obtidos por Veiga (2013).

Tabela 2.

Consistência interna (Alfa de Cronbach) da EAE-E4D

\begin{tabular}{lccc}
\hline Dimensões & $\begin{array}{c}\text { Presente } \\
\text { estudo }\end{array}$ & $\begin{array}{c}\text { Veiga } \\
(2013)\end{array}$ & $\mathrm{N}^{\circ}$ de itens \\
\hline Cognitiva & .702 & .768 & 5 \\
Afectiva & .906 & .823 & 5 \\
Comportamental & .758 & .706 & 5 \\
Agenciativa & .883 & .855 & 5 \\
Total & .852 & .828 & 20 \\
\hline
\end{tabular}

No estudo da análise fatorial de Fernandes et al. (2016) também realizado com estudantes de ensino superior, os valores de consistência interna (com 17 itens) foram, no geral semelhantes, apesar de sensivelmente mais baixos (0.698 na dimensão cognitiva, 0.813 na dimensão afetiva, 0.439 na dimensão comportamental e 0.797 na escala total), com exceção da dimensão agenciativa em que o valor foi ligeiramente superior $(0.882)$.

\section{Considerações finais}

Os resultados obtidos neste estudo permitem considerar que a utilização da EAE-E4D é igualmente adequada para avaliar o envolvimento dos estudantes de ensino superior. Concretamente, os resultados replicam os obtidos no estudo da escala original, relativamente à validade interna e à fidelidade da escala. Porém, é de ressalvar a importância de continuar estudos que incidam sobre a validação externa do instrumento e sobre a análise fatorial confirmatória.

\section{Referências}

Appleton, J. J., Christenson, S. L., \& Furlong, M. J. (2008). Student engagement with school: Critical conceptual and methodological issues of the construct. Psychology in the Schools, 45(5), 369-386. http://doi.org/10.1002/pits.20303

Astin, A. (1984). Student Involvement: A Developmental Theory for Higher Education. Journal of College Student Development, 40(5), 518-529. http://doi.org/10.1016/0263- 
Brophy, J. (1983). Conceptualizing student motivation. Educational http://doi.org/10.1080/00461528309529274

Fernandes, H. M. R., Caldeira, S., Silva, O. D. L., \& Veiga, F. H. (2016). Envolvimento dos Alunos no Ensino Superior: Um estudo com a Escala Quadridimensional de Envolvimento dos Alunos na Escola (EAE-E4D). In F. H. Veiga (Ed.), Envolvimento dos Alunos na Escola: Perspectivas da Psicologia da Educação - Motivação para o Desenpenho Académico / StudentsEngagement in School: Perspectives of Psychology and Education Motivation for academic Performance (pp. 47-76). Lisboa: Instituto de Educação da Universidade de Lisboa.

Finn, J. D. (1989). Withdrawing From School. Review of Educational Research, 59(2), 117-142. http://doi.org/10.3102/00346543059002117

Finn, J. D., \& Zimmer, K. S. (2012). Student Engagement: What is it? Why does it matter? In S. L. Christenson, A. L. Reschly, \& C. Wylie (Eds.), Handbook of Research on Student Engagement (pp. 97-131). New York: Springer. http://doi.org/10.1007/978-1-4614-2018-7

Fredricks, J. A., Blumenfeld, P. C., \& Paris, A. H. (2004). School Engagement: Potential of the Concept, State of the Evidence. Review of Educational Research, 74(1), 59-109. http://doi.org/10.3102/00346543074001059

Fredricks, J. A., \& McColskey, W. (2012). The measurement of student engagement: A comparative analysis of various methods and student self-report instruments. In S. L. Christenson, A. L. Reschly, \& C. Wylie (Eds.), Handbook of Research on Student Engagement. http://doi.org/10.1007/978-1-46142018-7

Fredricks, J. A., McColskey, W., Meli, J., Mordica, J., Montrosse, B., \& Mooney, K. (2011). Measuring Student Engagement in Upper Elementary through High School: A Description of 21 Instruments. Issues \& Answers. REL 2011 - No. 098. Regional Educational Laboratory Southeast.

Martins, C. (2011). Manual de Análise de Dados Quantitativos com recurso ao IBM SPSS: Saber, decidir, fazer, interpretar e redigir. (Psiquilibr). Braga.

Pintrich, P. R. (2003). Motivation and Classroom Learning. In I. B. Weiner, J. A. Schinka, \& W. F. Velicer (Eds.), Handbook of Psychology (p. Two:6: 103-122). John Wiley \& Sons. http://doi.org/10.1002/0471264385.wei0706

Reeve, J. (2012). A self-determination theory perspective on student engagement. In S. L. Christenson, A. L. Reschly, \& C. Wylie (Eds.), Handbook of Research on Student Engagement (pp. 149-172). New York: Springer. http://doi.org/10.1007/978-1-4614-2018-7

Reeve, J. (2013). How students create motivationally supportive learning environments for themselves: The concept of agentic engagement. Journal of Educational Psychology, 105(3), 579-595. http://doi.org/10.1037/a0032690
Reeve, J., \& Tseng, C. M. (2011). Agency as a fourth aspect of students' engagement during learning activities. Contemporary Educational Psychology, 36(4), 257-267. http://doi.org/10.1016/j.cedpsych.2011.05.002

Reschly, A. L., \& Christenson, S. L. (2012). Jingle, Jangle, and Conceptual Haziness: Evolution and Future Directions of the Engagement Construct. In S. L. Christenson, A. L. Reschly, \& C. Wylie (Eds.), Handbook of Research on Student Engagement (pp. 3-19). New York: Springer. http://doi.org/10.1007/978-1-4614-2018-7

Schaufeli, W. B., Salanova, M., Bakker, A. B., \& Gonzales-Roma, V. (2002). The Measurement of Engagement and Burnout: A two sample confirmatory Factor Analytic Approach. Journal of Happiness Studies, 3, 71-92. http://doi.org/10.1023/A:1015630930326

Skinner, E. A., Kindermann, T. A., \& Furrer, C. J. (2008). A Motivational Perspective on Engagement and Disaffection: Conceptualization and Assessment of Children's Behavioral and Emotional Participation in Academic Activities in the Classroom. Educational and Psychological Measurement. http://doi.org/10.1177/0013164408323233

Trowler, V. (2010). Student engagement literature review. Retrieved March 24, 2015, from http://americandemocracy.illinoisstate.edu/documents /democratic-engagement-white-paper-2_13_09.pdf

Tuckman, B. W. (2012). Manual de investigação em educação (4a Edição). Lisboa: Fundação Calouste Gulbenkian.

Veiga, F. H. (2013). Envolvimento dos Alunos na Escola: Elaboração de uma nova Escala de Avaliação. International Journal of Developmental and Educacional Psychology, 1(1), 441-450.

Veiga, F. H., Festas, I., Taveira, C., Galvão, D., Janeiro, I., Conboy, J., ... Nogueira, J. (2012). Envolvimento dos Alunos na Escola: Conceito e Relação com o Desempenho Académico — Sua Importância na Formação de Professores. Revista Portuguesa de Pedagogia, 46(2), 31-47.

Veiga, F. H., Galvão, D., Almeida, A., Carvalho, C., Janeiro, I., Nogueira, J., ... Caldeira, S. (2012). Student's Engagement in School: a Literature Review. In L. Chova, A. Martínez, \& I. Torres (Eds.), Atas da 5th International Conference of Education, Research, and Innovation- ICERI 2012 (pp. 13361344). Madrid.

Veiga, F. H., Reeve, J., Wentzel, K., \& Robu, V. (2014). Assessing students' engagement: A review of instruments with psychometric Qualities. In F. H. Veiga (Ed.), Envolvimento dos Alunos na Escola: Perspetivas Internacionais da Psicologia e Educação / Students' Engagement in School: International Perspectives of Psychology and Education (pp. 3857). Lisboa: Instituto de Educação, Universidade de Lisboa.

Weiner, B. (1985). An attributional theory of achievement motivation and emotion. Psychological Review, 92(4), 548-573. http://doi.org/10.1037/0033295X.92.4.548 
COVAS, VEIGA

\section{Nota}

O presente estudo insere-se num outro mais amplo, tese de doutoramento com o título "Envolvimento na Escola e Saúde Mental dos Estudantes de Ensino Superior", realizado pela primeira autora com a orientação do Professor Feliciano H Veiga. 